\title{
Steroid Responsive Parenchymal Lung Involvement and Acute Renal Failure in a Patient with Relapsing Polychondritis
}

\author{
Aman Sharma, Susheel Kumar, Palvi Goyal, Arjun D Law, Anupam Lal, Surjit Singh
}

\begin{abstract}
Relapsing polychondritis is a multisystem disorder affecting cartilaginous structures like ear, nose, laryngotracheal tree and proteogylcan rich structures like eyes. Kidney involvement has also been reported in the absence of associated conditions like SLE and vasculitides but parenchymal lung involvement has not been reported in the English literature. One such case of relapsing polychondritis who had parenchymal lung involvement with respiratory failure, renal failue and pancytopenia is described. All these complications recovered with institution of corticosteroids.
\end{abstract}

Keywords: Relapsing polychondritis, Lung involvement, Acute renal failure.

How to cite this article: Sharma A, Kumar S, Goyal P, Law AD, Lal A, Singh S. Steroid Responsive Parenchymal Lung Involvement and Acute Renal Failure in a Patient with Relapsing Polychondritis. J Postgrad Med Edu Res 2012;46(4):198-201.

Source of support: Nil

Conflict of interest: None declared

\section{INTRODUCTION}

Relapsing polychondritis (RP) is a multi-system disorder characterized by recurrent inflammation and destruction of cartilages of the external ear, nose, larynx, trachea and major bronchi and also of proteoglycan rich structures like eyes and heart. ${ }^{1-4}$ Men and women are affected equally. Respiratory tract involvement, though uncommon at presentation, occurs in up to $50 \%$ of patients with relapsing polychondritis sometime during the course of their illness. Primary parenchymal lung involvement is extremely rare manifestation of this disorder and has only been described in a case published in Japanese language. ${ }^{4}$ This has not been described in English language. Renal involvement is also uncommomly seen in these patients. ${ }^{1,4}$ We report a case of relapsing polychondritis with primary lung parenchymal involvement possibly in the form of organizing pneumonia, renal involvement and pancytopenia. All these manifestations showed dramatic response to institution of high dose steroid therapy.

\section{CASE REPORT}

A 58-year-old man had history of fever, fatigue, redness of eyes and generalized body aches of three and half years duration. He also had ear cartilage involvement in the form of tenderness along with redness of both the ears sparing the lobules. A diagnosis of relapsing polychondritis was made according to the diagnostic criteria suggested by McAdam et al and modified by Damiani and Levine. ${ }^{1,6} \mathrm{He}$ was started on prednisolone and azathioprine. His symptoms improved with treatment. During follow-up, he developed bicytopenia in the form of anemia and leukopenia. A possibility of drug induced cytopenia was entertained and azathioprine was stopped and he was continued only on low dose prednisolone.

This time he presented with recurrence of fever, easy fatigubility and exertional shortness of breath. Physical examination revealed pallor and hepatosplenomegaly. Investigations revealed hemoglobin of $6.1 \mathrm{gm} / \mathrm{dl}$, total leuko-

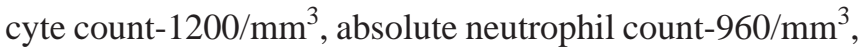
platelet count-49 $\times 10^{3} / \mu \mathrm{l}$ and ESR-71mm/ 1st hour. At presentation serum creatinine was $1.04 \mathrm{mg} / \mathrm{dl}$ and other biochemical parameters were also normal. Chest X-ray, ECG, urine routine and microscopic examination were also normal initially. Blood culture grew Pseudomonas aeruginosa. He was started on antibiotics according to sensitivity pattern. Patient initially responded for a few days but started developing fever again after a few days. CECT chest and abdomen was done as a part of fever workup. It revealed enlarged liver and spleen with wedge shaped hypodense areas in spleen; lung parenchyma and other organs system examination were normal. 2D-Echo was normal and did not reveal any evidence of infective endocarditis. Bone marrow aspiration and biopsy showed hypercellular marrow spaces with a few atypical cells in few of marrow spaces besides increase in fibrosis. As patient continued to run fever and neutropenia persisted, empirical antifungal (Conventional amphotericin B) was initiated. One day later he developed oliguria and rise in blood urea and serum creatinine. He was started on alternate day hemodialysis and liposomal amphotericin B was started in place of conventional amphotericin B. Gradually the hemoglobin decreased to5.6 gm/dl, total leukocyte count decrease to $2200 / \mathrm{cu} \mathrm{mm}$, and platelet count decreased to $23,000 / \mathrm{cu} \mathrm{mm}$ and the serum creatinine increased to $12.8 \mathrm{mg} / \mathrm{dl}$. His ANA was negative and flow cytometry for CD 58, CD 55, CD 59 and CD 11b done to look for PNH was normal. Serum ferritin was more than $1200 \mathrm{ng} / \mathrm{ml}$ suggesting an increase as an acute phase reactant. 
Patient continued to run fever; pancytopenia persisted though his urine output started improving. During this time while on alternate day hemodialysis and improving urine output, he acutely developed shortness of breath at rest. Physical examination revealed diffuse crepitation over bilateral inter-infrascapular and infra-axilliary areas. Jugular venous pressure was not raised. Cardiac examination revealed only tachycardia. Chest X-ray showed diffuse infiltrates in bilateral lung fields with no specific predilection for any side or zones. ECG did not show any ischemic changes. HRCT chest showed septal thickening in bilateral upper lobes and consolidation along with ground glass appearance in bilateral lower lung fields (Fig. 1). He developed respiratory failure and was put on noninvasive ventilation. Patient was still febrile despite receiving broad spectrum antibiotics and antifungal therapy. All this while, there was no evidence of cartilaginous inflammation in the form of ear/ nose or throat pain and is was after 1 month of extensive evaluation, investigation and treatment that he developed pain in the ears along with blurring of vision. Examination revealed evidence of scleritis and inflamed ear cartilages with sparing of lobules. Now a possibility of active disease causing lung, renal and hematological abnormality was considered and he was given five daily pulses of methylprednisolone (1 gm each) followed by oral steroids $(1 \mathrm{mg} / \mathrm{kg}$ prednisolone initially tapered rapidly after 4 weeks. He had a dramatic response with subsidence of fever and signs of cartilaginous inflammation. Shortness of breath also improved over next few days. A repeat HRCT chest showed showed near complete resolution of lung abnormalities

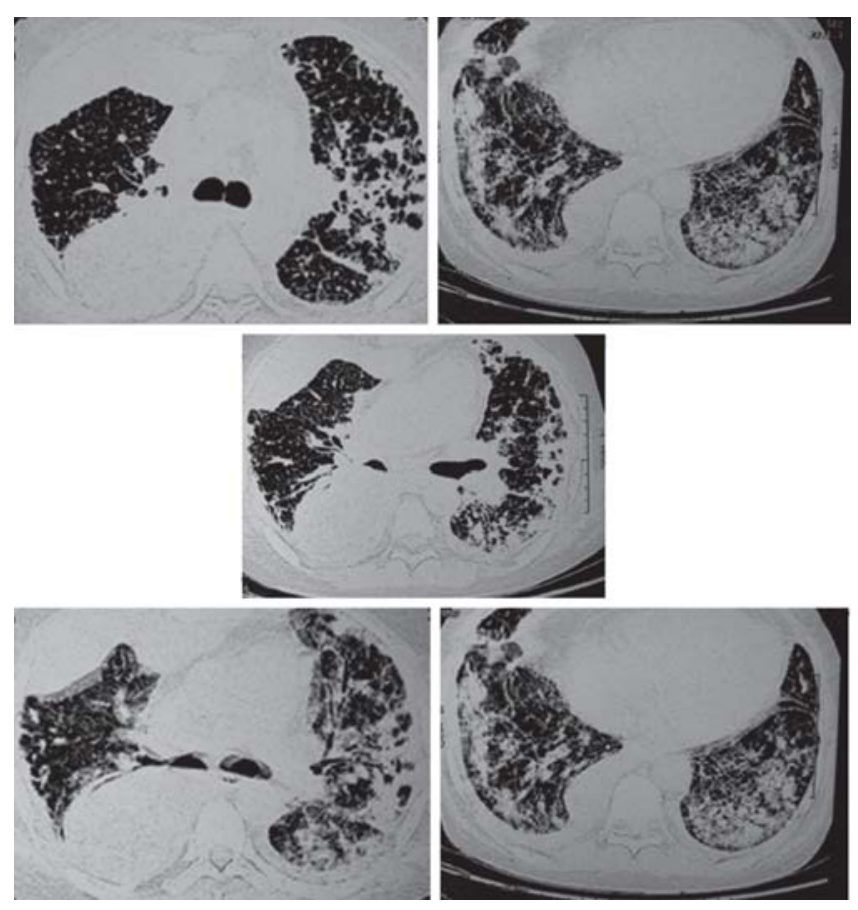

Fig. 1: Computerized tomography pictures showing septal thickening with extensive patchy ground glass opacities
(Fig. 2). At the time of discharge, Blood counts improved significantly with hemoglobin of $9.6 \mathrm{gm} / \mathrm{dl}$, serum creatinine comin down to $2.6 \mathrm{mg} / \mathrm{dl}$. On a follow-up, 1 month after the discharge, his hemoglobin was $9 \mathrm{gm} / \mathrm{dl}$, total leukocyte count was 4000/cu mm and serum creatinine had become normal.

\section{DISCUSSION}

Respiratory tract involvement in the form of laryngotracheobronchial disease, which can be complicated by laryngeal, tracheal, and/or bronchial obstruction, is seen in approximately $50 \%$ of the patients with RP. ${ }^{1,4}$ Primary lung parenchymal involvement is extremely rare. Till date to the best of our knowledge there is single case reported in Japenese journal with primary parenchymal lung involvement. In this case report, a 60-year-old male had features of cartilage inflammation, conjunctivitis, episcleritis and deafness as well. He later developed spontaneous pneumothorax first on the left side and then on the right side. CT chest showed bilateral pneumothoraces, many bilateral bullae and emphysematous changes. There was also ground glass shadow, consolidation, honeycombing and decreased pulmonary volume. ${ }^{4}$ Our patient had septal thickening in bilateral upper lobes and consolidation along with ground glass appearance in bilateral lower lung fields. Most importantly all these lung abnormalities responded dramatically to high dose steroid therapy indicating these to be autoimmune in nature. Severe pancytopenia and advanced renal failure precluded any attempt to get a tissue diagnosis either by open lung biopsy or transbronchial lung biopsy.

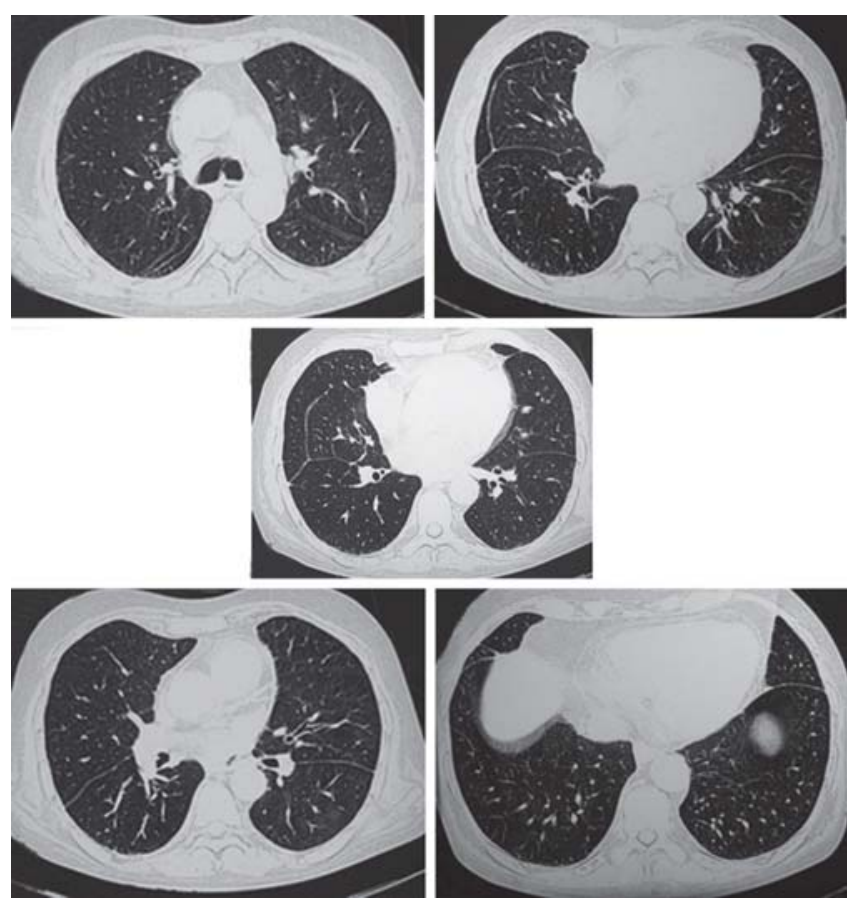

Fig. 2: Computerized tomography pictures showing almost complete resolution of the lung opacities after institution of methylprednisolone 
Davison et al in 1983 described a clinicopathologic entity and named it cryptogenic organizing pneumonitis (COP). ${ }^{7}$ This disease was described under the term bronchiolitis obliterans organizing pneumonia (BOOP) in 1985 by Epler et al. ${ }^{8}$ This type of organizing pneumonia has been reported in association with connective tissue disordes and sometimes viral infections. ${ }^{9} \mathrm{CT}$ shows areas of airspace consolidation in $90 \%$ of patients with COP. Subpleural or peribronchial distribution is seen in up to 50\% of cases. The lower lung zones are more frequently involved. Ground glass attenuation is present in about $60 \%$ of cases, usually associated with lung consolidation. ${ }^{9-11}$ Rarely, pleural effusion may be seen in these patients. Our index patient had similar abnormality in HRCT chest. It showed septal thickening in bilateral upper lobes and consolidation along with ground glass appearance in bilateral lower lung fields along with pleural effusion. Substantial number of patients with COP demonstrates radiographic improvement with steroid. There is complete clearing of lung abnormalities in most of these patients who respond to steroids. ${ }^{9}$ Our patient had also shown complete resolution of lung abnormalities in repeat CT chest. We thus, propose organizing pneumonia as new primary parenchymal manifestation of relapsing polychondritis.

Renal disease is an uncommon manifestation of RP but may result in patient's mortality. Deranged renal function has been reported in approximately $10 \%$ of RP patients. Kidney involvement is caused by either primary renal parenchymal lesions or an underlying systemic vasculitis or associated connective tissue disorder. The most common histologic feature seen in these patients is mild mesangial proliferation on renal biopsy. Other findings include necrotizing glomerulonephritis with crescent formation, glomerulosclerosis, IgA nephropathy, and tubulointerstitial nephritis. ${ }^{1-4,12,13}$ Our patient developed renal dysfunction during this presentation. Amphotericin induced renal toxicity seems very unlikely as patient developed renal shutdown almost immediately after the initiation of amphotericin-B infusion. Importantly renal dysfunction responded to high dose methylprednisolone indicating renal pathology being autoimmune in origin. It seems that it was a sheer coincidence that initiation of amphotericin-B infusion and development of renal dysfunction developed simultaneously. Renal biopsy was not done as patient had concurrent thrombocytopenia.

Various forms of hematological disorders, e.g. myelodysplastic syndrome (MDS), non-Hodgkin's lymphoma (NHL), Hodgkin's lymphoma, hemolytic anemia and monoclonal-gammopathy have been described RP. ${ }^{14-16}$ Among these, concurrence of RP with MDS is relatively more frequent. MDS can precede, occur simultaneously or develop subsequently. Our patient had hematological abnormalities in the form of cytopenias and bone marrow showed hypercellular marrow spaces and few atypical cells. Hypercellular marrow spaces on examination and response of cytopenias to steroids leads us to believe that autoimmune process resulting in peripheral destruction of cell lines was responsible for cytopenias. Though explaining presence of atypical cells in marrow is difficult.

This case demonstrates that primary parenchymal involvement can be seen rarely in these patients. Renal involvement though uncommon may be seen in a patient with an active multi-system disease as seen in this case. These kind of uncommon but organ and life-threatening manifestations of RP can be treated with steroids with good results.

\section{REFERENCES}

1. McAdam LP, O’Hanlan MA, Bluestone R, Pearson CM. Relapsing polychondritis: Prospective study of 23 patients and a review of the literature. Medicine 1976;55:193-215.

2. Sharma A, Bambery P, Wanchu A, Sharma YP, Panda N, Gupta AK, et al. Relapsing polychondritis in North India: A report of ten patients. Scand J Rheumatol 2007;36:462.

3. Sharma A, Mittal T, Kumar S, Law AD, Wanchu A, Mahajan R, et al. Successful treatment of aortic root dilatation in a patient with relapsing polychondritis. Clin Rheumatol 2010 Apr 13. [Epub ahead of print]

4. Letko E, Zafirakis P, Baltatzis S, Voudouri A, Livir-Rallatos C, Stephen Foster C. Relapsing polychondritis: A Clinical Review Semin Arthritis Rheum 31:384-95.

5. Wu S, Sagawa M, Suzuki S, Kumagai-Braesch, Sato M, Kondo T. Pulmonary fibrosis with intractable pnemothorax: New pulmonary manifestation of relapsing polychondritis. Tohoku J Exp Med 2001;194:191-95.

6. Damiani JM, Levine HL. Relapsing polychondritis-report of 10 cases. Laryngoscope 1979;89:929-46.

7. Davison AG, Heard BE, McAllister WAC, Turner-Warwick ME. Cryptogenic organizing pneumonitis. Q J Med 1983;207:382-94.

8. Epler GR, Colby TV, McLoud TC, Carrington CB, Gaensler EA. Bronchiolitis obliterans organizing pneumonia. N Engl J Med 1985;312:152-58.

9. American Thoracic Society; European Respiratory Society. American Thoracic Society/European Respiratory Society International Multidisciplinary Consensus Classification of the Idiopathic Interstitial Pneumonias. This joint statement of the American Thoracic Society (ATS), and the European Respiratory Society (ERS) was adopted by the ATS board of directors, June 2001 and by the ERS Executive Committee, June 2001. Am J Respir Crit Care Med 2002;165:277-304.

10. Lee KS, Kullnig P, Hartman TE, Muller NL. Cryptogenic organizing pneumonia: CT findings in 43 patients. Am J Roentgenol 1994;162:543-46.

11. Müller NL, Staples CA, Miller RR. Bronchiolitis obliterans organizing pneumonia: CT features in 14 patients. Am J Roentgenol 1990;154:983-87.

12. Neild GD, Cameron JS, Lessof MH, Ogg CS, Turner DR. Relapsing polychondritis with crescentic glomerulonephritis. Br Med J 1977;1:743-45. 
13. Isaak BL, Liesegang TJ, Michet CJ Jr. Ocular and systemic findings in relapsing polychondritis. Ophthalmology 1986;93: 681-89.

14. Heo SW, Cho KH, Ryu JI, Chung SH, Kim CG, Kim SG, et al. A case of relapsing polychondritis associated with myelodysplastic syndrome with erythroid hypoplasia/aplasia. Korean J Intern Med 2003;18:251-54.

15. Banerjee SS, Morris DP, Rothera MP, Routledge RC. Relapsing polychondritis associated with monoclonal gammopathy in a patient with myelodysplastic syndrome. J Laryngol Otol 2001; 115:482-84.

16. Yanagi T, Matsumura T, Kamekura R, Sasaki N, Hashino S. Relapsing polychondritis and malignant lymphoma: Is polychondritis paraneoplastic? Arch Dermatol 2007 Jan;143(1): 89-90.

\section{ABOUT THE AUTHORS}

\section{Aman Sharma (Corresponding Author)}

Assistant Professor, Department of Internal Medicine Postgraduate Institute of Medical Education and Research Chandigarh, India, Phone: 91-172-2756681, Fax: 91-172-2744401 e-mail: amansharma74@yahoo.com

\section{Susheel Kumar}

Assistant Professor, Department of Internal Medicine, Postgraduate Institute of Medical Education and Research, Chandigarh, India

\section{Palvi Goyal}

Senior Resident, Department of Internal Medicine, Postgraduate Institute of Medical Education and Research, Chandigarh, India

\section{Arjun D Law}

Senior Resident, Department of Internal Medicine, Postgraduate Institute of Medical Education and Research, Chandigarh, India

\section{Anupam Lal}

Associate Professor, Department of Radiodiagnosis and Imaging Postgraduate Institute of Medical Education and Research, Chandigarh India

\section{Surjit Singh}

Professor, Department of Internal Medicine, Postgraduate Institute of Medical Education and Research, Chandigarh, India 